\title{
503-COMPARATIVE STUDY OF THE ELECTROCHEMICAL BEHAVIOUR OF GLUCOSE AND OTHER COMPOUNDS OF BIOLOGICAL INTEREST
}

\author{
M.F.L. de MELE, H.A. VIDELA and A.J. ARVÍA \\ Instituto de Investigaciones Fisicoquimicas Teóricas y Aplicadas (INIFTA). Casilla de Correo I6, Sucursal \\ 4-(1900) La Plata (Argentina)
}

(Manuscript received December 21st 1981)

\section{SUMMARY}

The electro-oxidation of glucose and related compounds was studied in phosphate buffered media using a smooth platinum working electrode at different temperatures. A comparative analysis of the electrochemical behaviour of glucose and gluconic acid was made. Different electrochemical responses were found in the hydrogen adatom and in the PtO layer formation potential regions. Potentiostatic and potentiodynamic measurements with inositol, fructose, xylose, arabinose and glucose-1-phosphate were also performed in an attempt to find a relation between the electrochemical behaviour and the structural characteristics of these molecules. Our results suggest that the hemiacetalic group of the glucose molecule seems to play an important role in the electro-oxidation process. The electro-oxidation order of the different carbohydrates and derivatives resembles to some extent the electrochemical conversion order already established for other organic compounds.

\section{INTRODUCTION}

Recently the electrochemical oxidation of glucose on platinum has been studied with special interest because of its importance for possible electrical devices to be used in living organisms. Thus, efforts were made to determine the possibility of utilizing dextrose as a fuel in an implantable biofuel cell useful as a power source for electronic devices of medical interest [1-6]. The possibility of developing a biological sensor of glucose level in relation to an artificial pancreas was also considered [7-14]. However, despite the practical applications of the electrochemical oxidation of glucose, knowledge of the kinetic mechanism of the reaction is far from complete. A critical review of the available literature related to this reaction shows that different mechanistic interpretations were postulated for the electro-oxidation of glucose. Thus, according to Rao and Drake the electro-oxidation of glucose on platinized platinum in phosphate buffered medium [15] yields gluconic acid as the only reaction product. The latter apparently inhibits the oxidation of dextrose through its adsorption on the electrode surface. A similar electrochemical behaviour 
has been observed by Yao et al. for glucose and gluconic acid [16] on platinum electrodes, the electro-oxidation of both substances involving the same rate-determining step. Coincidently, Giner and Malachesky found that glucose and gluconic acid present similar oxidation behaviour at low potentials, both yielding a chemisorbed product which was tentatively identified as reduced $\mathrm{CO}_{2}$ [17]. The potentiostatic electrochemical oxidation of glucose within the $-0.2 \mathrm{~V}$ to $0.2 \mathrm{~V}$ (s.c.e.) potential region [18] exhibits a continuous decrease of current which was attributed to the progressive deactivation of the platinum electrode. The number of charges transferred per reacting molecule varied with time and potential, in the range between 4 and 20 . The current decay at a constant potential suggests a change in the electro-oxidation mechanism with time, and opened the possibility that gluconic acid is not the only reaction product. Potentiostatic [18] and potentiodynamic [19] studies of the reaction in a Krebs-Ringer medium saturated with a mixture $\mathrm{CO}_{2}-\mathrm{N}_{2}$, indicated the inhibition of glucose adsorption in the presence of $\mathrm{CO}_{2}[20]$.

According to Skou, the presence of gluconic acid, produced through the hydrolysis of glucono $\delta$-lactone in glucose solutions inhibits the electrooxidation of glucose in the hydrogen adatom potential range [21,22]. This was explained through a reaction mechanism comprising an intermediate glucose adsorption pathway [21]. It was assumed that the adsorbed glucose rearranges in the corresponding enediol which can diffuse into the bulk of the solution or become oxidized in a two-electron process [23]. On the other hand, through the calculation of the accumulation of charge at the electrode, the closed form of glucose was excluded as the main reactive species [17]. Notwithstanding, the hemiacetalic group of glucose was associated with the reactive centre [24] and glucono- $\delta$-lactone was identified as a primary reaction product through mass spectroscopic analysis [25]. In the potential region between $-0.6 \mathrm{~V}$ and $-0.3 \mathrm{~V}$ (s.c.e.) the electro-oxidation mechanism was also explained through the glucose adsorption which was favoured by the presence of hydrogen adatoms [24]. The dehydrogenation of the $C_{1}$-atom adjacent to the hemiacetalic $\mathrm{OH}$-group was proposed as the rate-determining step. In the potential region within -0.3 to $+0.1 \mathrm{~V}$ (s.c.e.) on a surface partially covered with the species electro-adsorbed in the region between $-0.6 \mathrm{~V}$ and $-0.3 \mathrm{~V}$, the electro-oxidation of dextrose apparently occurs through two consecutive dehydrogenation steps [25].

The ionic composition, the buffer capacity of the solution, and the ionic strength modify the electrochemical behaviour of glucose $[20,26,27]$. This explains some of the differences observed in the $U / I$ profiles obtained in diluted [26-28] and concentrated buffers [22-27]. However, in previous works little attention has been paid to the importance of the solution side of the electrochemical double layer. Recently, the influence of the buffer capacity and solution $\mathrm{pH}$ on the electro-oxidation of glucose on platinum was reported $[20,27,29]$.

$D$-glucose exists in aqueous solutions as two different isomeric forms ( $\alpha$ - and $\beta$-glucose) the interconversion of these isomers, occurring through the open-chain form ( $\gamma$-glucose). The ring isomers are formed by the reaction of the alcoholic hydroxyl group at carbon atom 5 with the aldehydic group at carbon atom 1. 
Whenever this reaction is produced a hemiacetalic structure is formed.

The principal oxidation product of D-glucose either in chemical or electrochemical reactions (gluconic acid), derives from oxidation of the aldehydic carbon atom of the hexose. The lactone ring form of gluconic acid (glucono- $\delta$-lactone) is formed as the first intermediate oxidation product in the case of $\alpha-, \beta$-glucose [24,25].

With the aim of comparing the electrochemical behaviour of glucose with those compounds of similar structure, but presenting five-member ring molecules (furanose form), results related to the electrochemical characteristics of arabinose and xylose are given in this paper. In addition, for study of the electrochemical behaviour of a cyclic structure of six carbon atoms, containing only $\mathrm{OH}$-groups, with neither hemiacetalic nor carbonyl forms, the $U / I$ profiles of glucose with the cyclohexane derivative alcohol (inositol) are compared. The importance of the hemiacetalic structure in the electro-oxidation of glucose is evaluated by comparing the electrochemical spectra of a monosaccharide and its derivatives where the aldehydic group has been either replaced by a carbonyl group at carbon atom 2 (fructose) or blocked by esterification with phosphate (glucose-1-phosphate).

\section{EXPERIMENTAL}

A conventional three-compartment Pyrex glass electrolysis cell was used. A smooth platinum wire (polycrystalline, specpure, Johnson Matthey) of $0.16 \mathrm{~cm}^{2}$ apparent area was used as working electrode. The platinum counter electrode also consisted of a Pt wire. A saturated calomel electrode properly shielded was used as reference. Experiments were carried out in nitrogen-saturated electrolyte solutions at $37^{\circ} \mathrm{C}$, and also at $30,44,54$ and $64^{\circ} \mathrm{C}$.

The electrolyte solutions were prepared from AR chemicals and triply distilled water. The phosphate buffer $\left(\mathrm{KH}_{2} \mathrm{PO}_{4}+\mathrm{K}_{2} \mathrm{HPO}_{4}\right)$, ionic strength $I=0.2 \mathrm{M}$ and $\mathrm{pH}=7.4$, was the main electrolyte solution. The total ionic strength was set at $1.0 \mathrm{M}$ by addition of $\mathrm{K}_{2} \mathrm{SO}_{4}$. Occasionally, $0.1 \mathrm{M} \mathrm{K}_{2} \mathrm{HPO}_{4}$ and $0.1 \mathrm{M} \mathrm{KH}_{2} \mathrm{PO}_{4}$ buffer solutions without the addition of $\mathrm{K}_{2} \mathrm{SO}_{4}$ were employed.

The working electrode was perturbated with different potential/time programs, including triangular sweeps (TPS) and combined functions (Fig. 1). Starting with a reproducible electrode surface, the perturbation program depicted in Fig. 1a was used on the basis of previous works [30,31]. To remove any trace of adsorbed organic species from the surface and to provide the electrode with a passive oxygen film, the potential of the working electrode was first stepped at $1.2 \mathrm{~V}$ and then at either 0.8 or $1.0 \mathrm{~V}$, to eliminate any molecular oxygen produced in the first step. Later, the potential was set at the electroadsorption potential $\left(U_{a d}\right)$ for a certain time $\left(t_{a d}\right)$, and subsequently a triangular sweep was applied towards the positive potential direction from $U_{a d}$ up to either 0.8 or $0.9 \mathrm{~V}$ and backwards to $U_{a d}$ to ensure complete electroreduction of the oxygen-containing film produced during the anodic sweep.

Occasionally, other perturbation programs were used. As is shown in Fig. 1b, after the two initial potential steps, a triangular potential sweep in the negative 


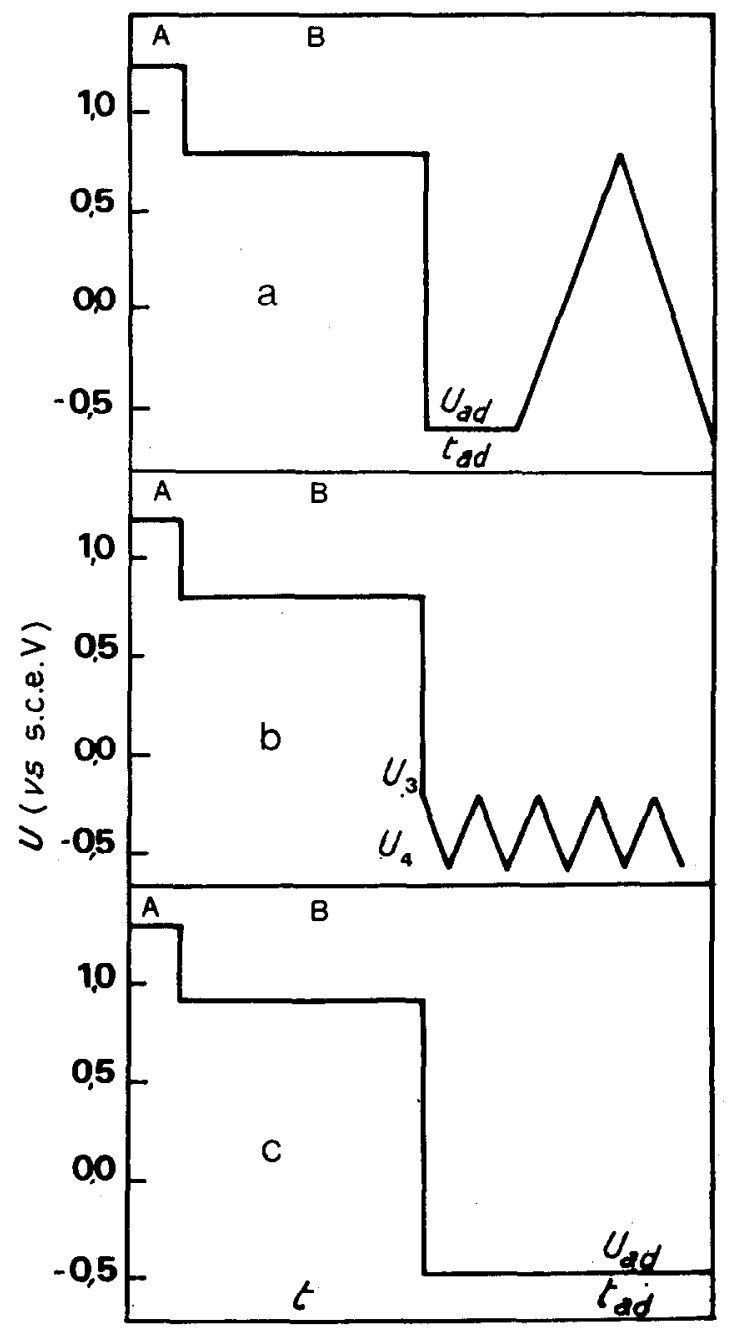

Fig. 1. Potential/time perturbation programs. (A) $10 \mathrm{~s}$; (B) $180 \mathrm{~s}$.

potential direction from a certain potential $U_{3}$ to $-0.6 \mathrm{~V}$ was applied in order to avoid electrode blockage by organic substances during the anodic sweep. On the other hand, potentiostatic $I$ versus time plots were performed at $U_{a d}$ after applying two potential steps at 1.2 and $0.8 \mathrm{~V}$ respectively, as described in Fig. Ic.

\section{RESULTS}

$U / I$ profiles run both in the presence of glucose and gluconic acid

In Fig. 2 the potentiodynamic $U$ versus $I$ profile corresponding to glucose electro-oxidation is shown. The display in the positive-going potential direction 


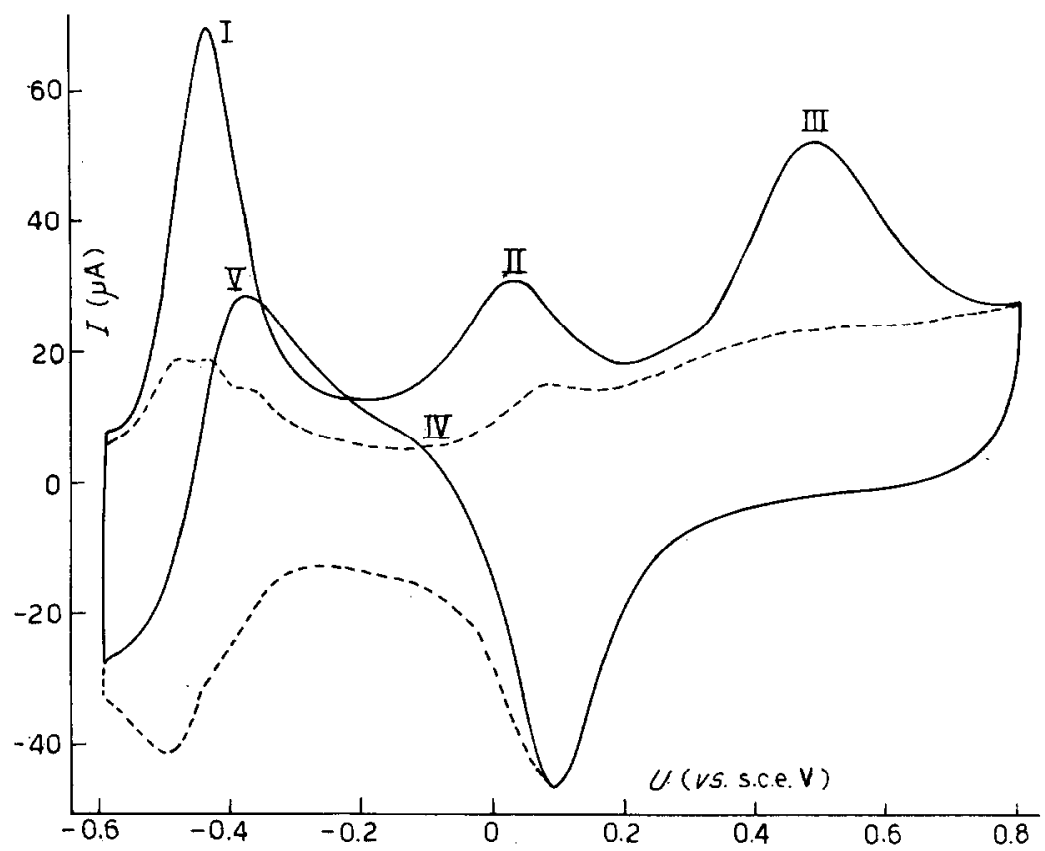

Fig. 2. Potentiodynamic $U / I$ profiles run in a $0.03 \mathrm{M}$ glucose solution (potential/time perturbation program shown in Fig. 1a, $U_{a d}=-0.6 \mathrm{~V}, t_{a d}=10 \mathrm{~s}$ ). Dotted line corresponds to the base electrolyte.

shows three anodic current peaks (I, II and III) which are specifically related to the presence of glucose in the electrolyte, and in the reverse potential scan a net anodic current peak (V) preceded by a wide hump (IV) located at the positive potential side of the O-electrodesorption current peak is recorded. A straightforward comparison of the $U$ versus $I$ display obtained in the presence of glucose with the blank, allows the evaluation of changes related to the Pt|electrolyte interphase as far as the $\mathrm{H}$ - and O-electroadsorption/electrodesorption processes are concerned. Peak $I$ is found just in the potential range of the $\mathrm{H}$-adatom electrosorption, the potential of peak II is located at the end of the double-layer region and peak III appears in the potential range where the $\mathrm{Pt}$ surface is fully covered with a monolayer of $\mathrm{O}$-species (PtO). On the other hand, both peak $\mathrm{V}$ and hump IV are recorded after the partial electrodesorption of the O-containing layer on Pt.

The $U$ versus $I$ profile run in the presence of gluconic acid (Fig. 3) as compared to that of glucose, in the potential range of peak III, exhibits a wide current plateau instead of a current peak. The relative peak heights are definitely dependent whether glucose or gluconic acid is present in the electrolyte solution. In addition, the potential of peak II is closely located in the region where the $\mathrm{Pt}(\mathrm{OH})_{a d}$ species are formed at the initial stage of O-electroadsorption on Pt [32]. Furthermore, peak V and hump IV which appear when glucose is present in the solution become a single small broad peak. 


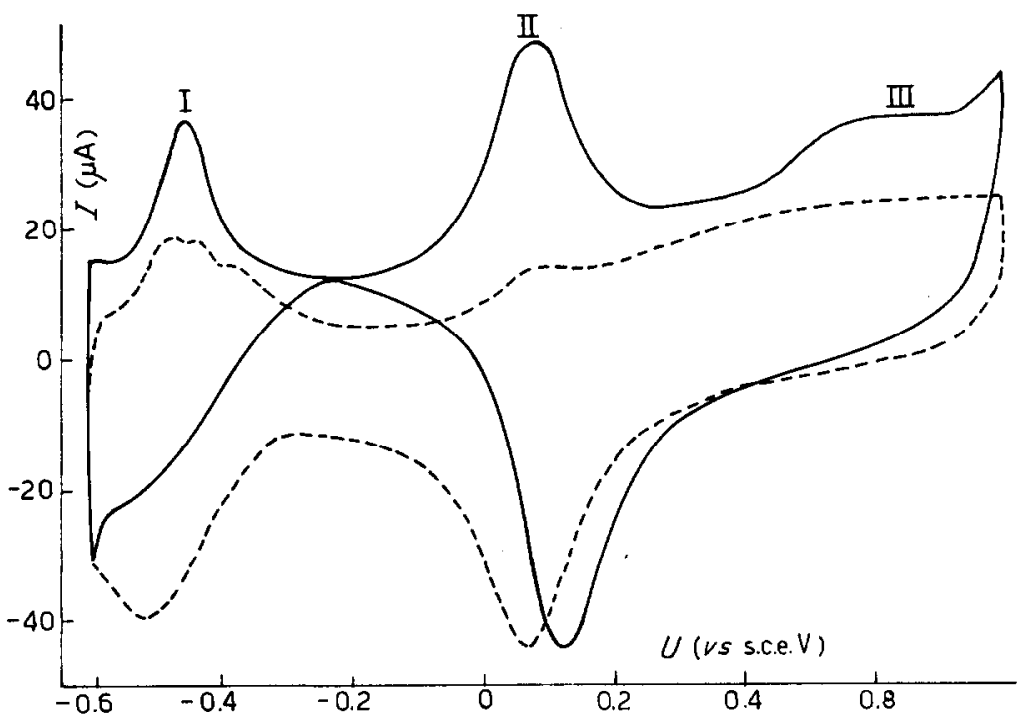

Fig. 3. Potentiodynamic $U / I$ profiles run in a $0.03 M$ gluconic acid solution (potential/time perturbation program shown in Fig. 1a, $U_{a d}=-0.6 \mathrm{~V}, t_{a d}=10 \mathrm{~s}$ ). Dotted line corresponds to the base electrolyte.

The temperature increase produces a remarkable change of the electrochemical characteristics of both glucose and gluconic acid electro-oxidation reactions (Figs. 4 and 5). Thus, the height of peak I decreases while both the heights and the charges of the current peaks located at more positive potential increase.

For glucose-containing solutions (Fig. 4) the increase of temperature produces the overlapping of peak $\mathrm{V}$ and hump IV, as well as a net increase of the anodic current. The $U / I$ profile in the positive-going potential direction, including the potential region of initiation of the $\mathrm{O}_{2}$ evolution, exhibits a decreasing polarization as the temperature increases, although the current associated with current peak $V$ moves in the opposite direction. This fact reveals that the electro-oxidation rate depends principally on the number of free sites available on the $\mathrm{Pt}$ surface. When the temperature increases, the O-electrodesorption is faster, and consequently during the potentiodynamic run the electrooxidation of glucose depends much more on the duration of the potential sweep than on the potential value itself. The same observations apply to the voltamperograms run in the negative potential-going direction in the presence of gluconic acid.

Current/time transients run at $U_{a d}$ with the potential program described in Fig. lc, also depend on the substance added to the electrolyte (Fig. 6). The electroadsorption of these substances takes place on the freshly electroreduced Pt surface. In the presence of glucose a remarkably high and sharp current peak involving an appreciable charge is observed. Conversely, the anodic charge involved during the current transient in the presence of gluconic acid is relatively small. In any case the charge obtained from the current versus time transient is, in principle, associated with the 


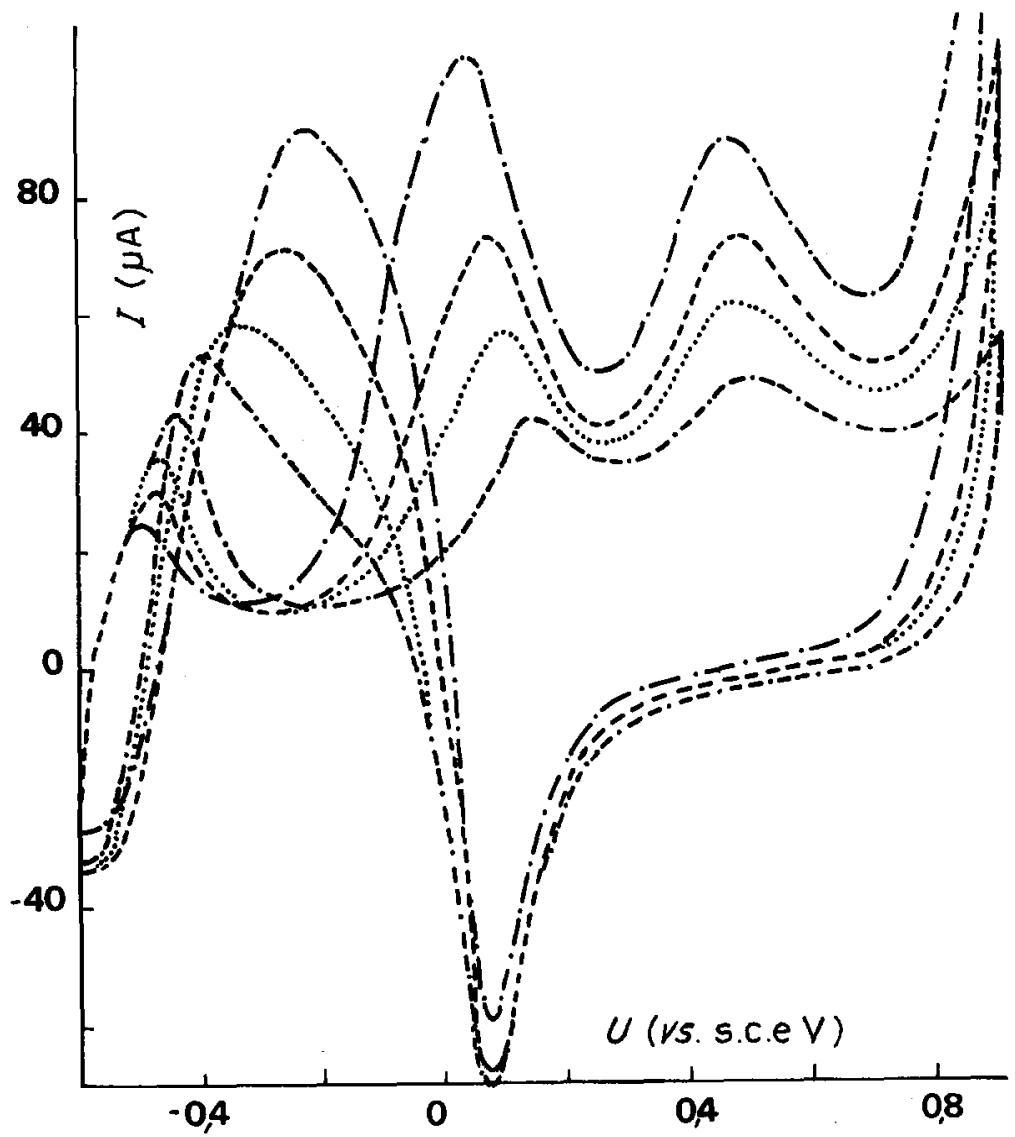

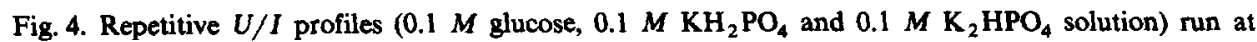
different temperatures: $(\cdot-\ldots) 34^{\circ} \mathrm{C}$; $(\cdots \cdots) 44^{\circ} \mathrm{C} ;(-\ldots . \cdot) 54^{\circ} \mathrm{C} ;(\cdot-\cdot) 64^{\circ} \mathrm{C}$.

electroadsorption of the organic molecule, but the remarkable difference found for the various substances should be attributed to a definite change in the corresponding electroadsorption process of each one of those molecules on $\mathrm{Pt}$.

The $U$ versus $I$ profiles corresponding to successive triangular sweeps in the hydrogen ad-atom potential region after pretreating the electrode, as shown in Fig. lb, exhibit some interesting features (Figs. 7 and 8). In the glucose-containing solutions during the first going scan, the large anodic charge is associated with its electro-oxidation (Fig. 7), whereas under the same experimental conditions in the presence of gluconic acid only a very small current peak is observed. Nevertheless, the H-adatom charge recorded during the successive potential sweeps decreases gradually both in the glucose- and gluconic acid-containing solutions. This suggests increasing blockage of the electrode surface by the electroadsorbed organic mole- 


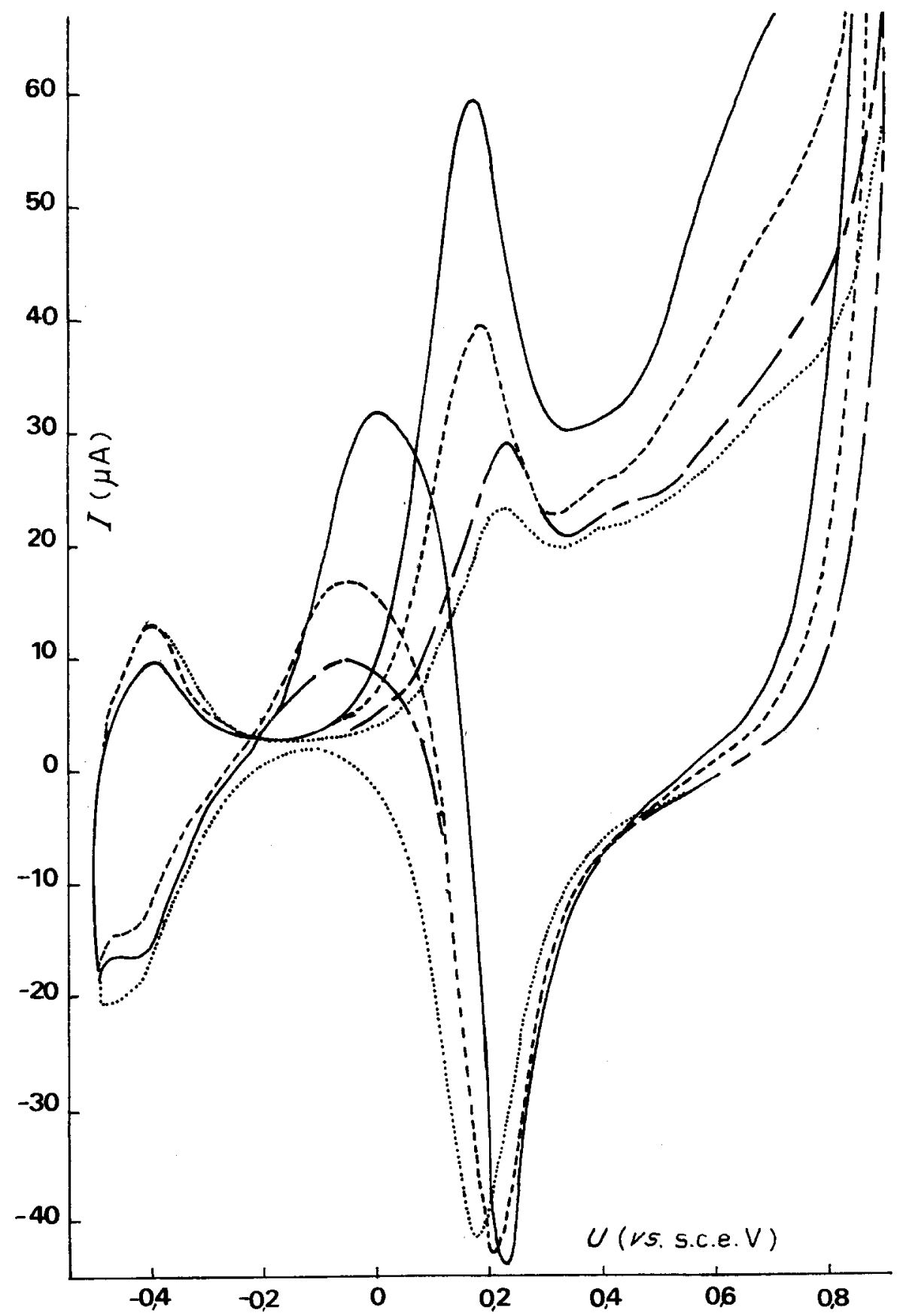

Fig. 5. Repetitive $U / I$ profiles ( $0.1 \mathrm{M}$ gluconic acid, $0.1 M \mathrm{KH}_{2} \mathrm{PO}_{4}$ and $0.1 M \mathrm{~K}_{2} \mathrm{HPO}_{4}$ solution) run at different temperatures: $(\cdots \cdots) 37^{\circ} \mathrm{C} ;(-\cdots--) 44^{\circ} \mathrm{C} ;(-\ldots .-) 54^{\circ} \mathrm{C} ;\left(\stackrel{-}{\square} 64^{\circ} \mathrm{C}\right.$. 


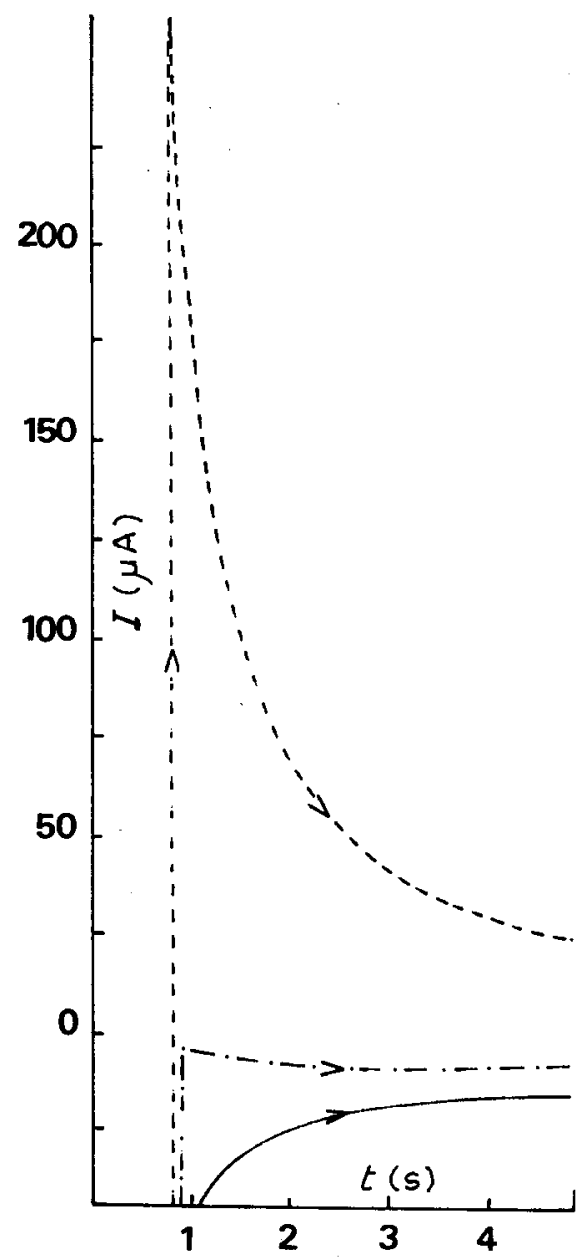

Fig. 6. Current transients recorded at $U_{a d}=-0.4 \mathrm{~V}$ (perturbation program shown in Fig. Ic) run in: (_- -) $0.03 \mathrm{M}$ glucose solution; (.-.-.) $0.03 \mathrm{M}$ gluconic acid solution; ( $\longrightarrow$ ) base electrolyte.

cules. In glucose solutions the height of current peak I depends on the time the electrode is held at $U_{a d}$ (Fig. 9) as well as on temperature (Fig. 4). At a constant temperature, the current associated with peak I decreases with increasing $t_{a d}$. Similar runs made with gluconic acid solutions (Fig. 10) show, in general, the same effect, although the current values in this case are lower than those recorded in the presence of glucose at the same temperature.

$U / I$ profiles run in the presence of glucono- $\delta$-lactone

Gluconolactone has been recently proposed as the only reaction product of the anodic process $[24,25]$. The structure of glucono- $\delta$-lactone is similar to that of 


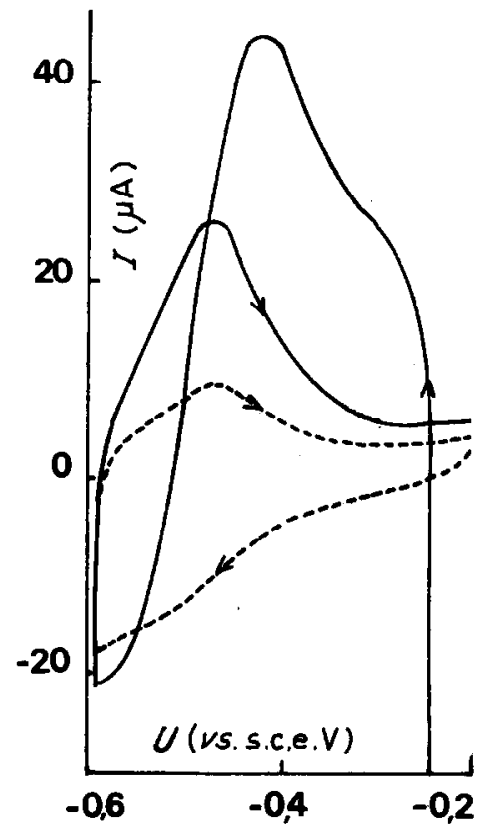

Fig. 7. Potentiodynamic $U / I$ profiles run in a $0.03 M$ glucose solution (potential/time perturbation program shown in Fig. $\left.1 \mathrm{~b}, U_{3}=-0.2 \mathrm{~V}, U_{4}=-0.6 \mathrm{~V}\right)$. First TPS $(-$

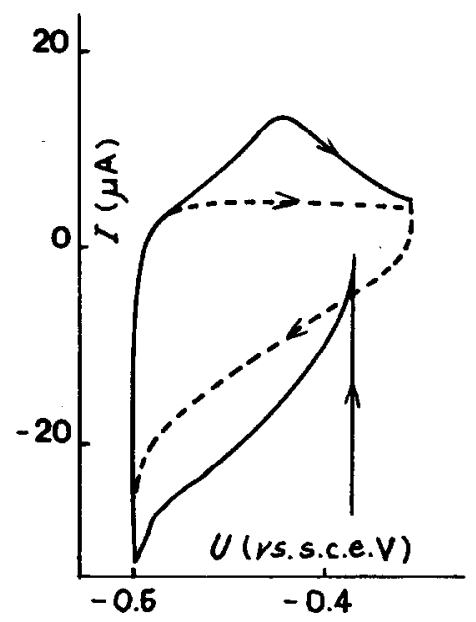

Fig. 8. Potentiodynamic $U / I$ profiles run in a $0.03 M$ gluconic acid solution (potential/time perturbation program shown in Fig. $\left.1 \mathrm{~b}, U_{3}=-0.3 \mathrm{~V}, U_{4}=-0.6 \mathrm{~V}\right)$. First TPS (- - ), second TPS $(---)$. 


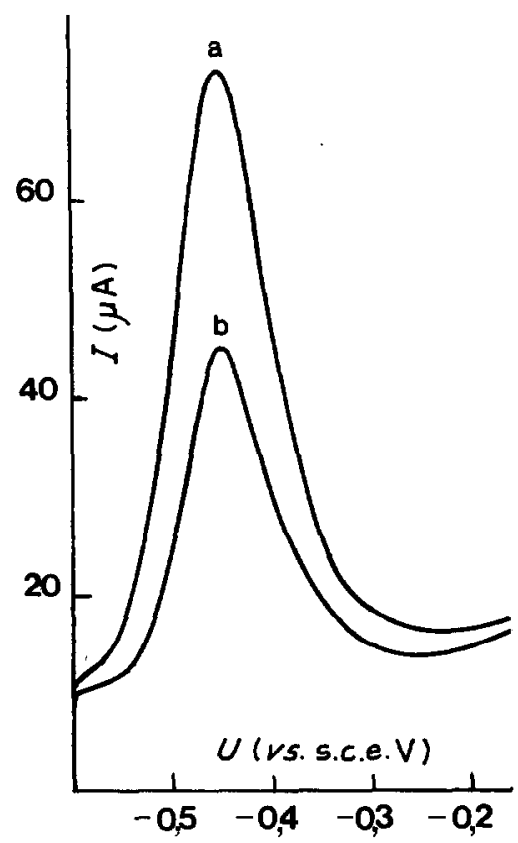

Fig. 9. Potentiodynamic $U / I$ profiles run in the anodic direction $(0.03 M$ glucose solution, potential/time perturbation program shown in Fig. $1 \mathrm{a}, U_{a d}=-0.6 \mathrm{~V}$ ); curve (a) $t_{a d}=10 \mathrm{~s}$; curve (b) $t_{a d}=60 \mathrm{~s}$.

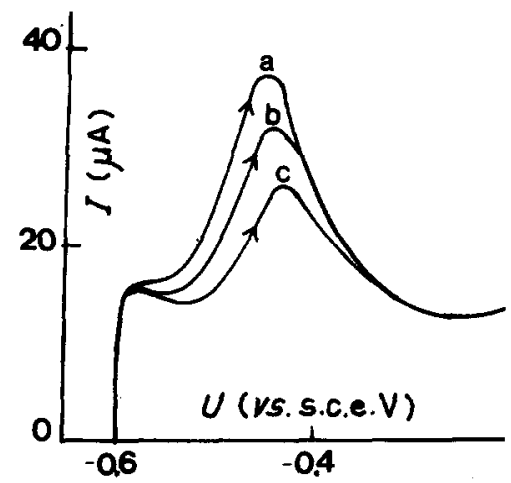

Fig. 10. Potentiodynamic $U / I$ profiles run in the anodic direction $(0.03 M$ gluconic acid solution, potential/time perturbation program shown in Fig. $1 \mathrm{a}, U_{a d}=-0.6 \mathrm{~V}$ ) curve (a) $t_{a d}=10 \mathrm{~s}$, curve (b) $t_{a d}=30 \mathrm{~s}$; curve (c) $t_{a d}=60 \mathrm{~s}$.

glucose, but it is unstable in aqueous solution, yielding through hydrolysis gluconic acid, according to the reaction:

$$
\mathrm{CH}_{2}(\mathrm{OH})-\mathrm{CH}-(\mathrm{CH}-\mathrm{OH})_{3}-\mathrm{CO}-\mathrm{O}+\mathrm{H}_{2} \mathrm{O} \rightarrow \mathrm{CH}_{2}(\mathrm{OH})-(\mathrm{CH}-\mathrm{OH})_{4}-\mathrm{CO}-\mathrm{OH}
$$

The rate constant of reaction (1) is $k=10^{-3} \mathrm{dm}^{3} / \mathrm{s}$ at $\mathrm{pH}=7.5$ and $20^{\circ} \mathrm{C}$ [33]. The 


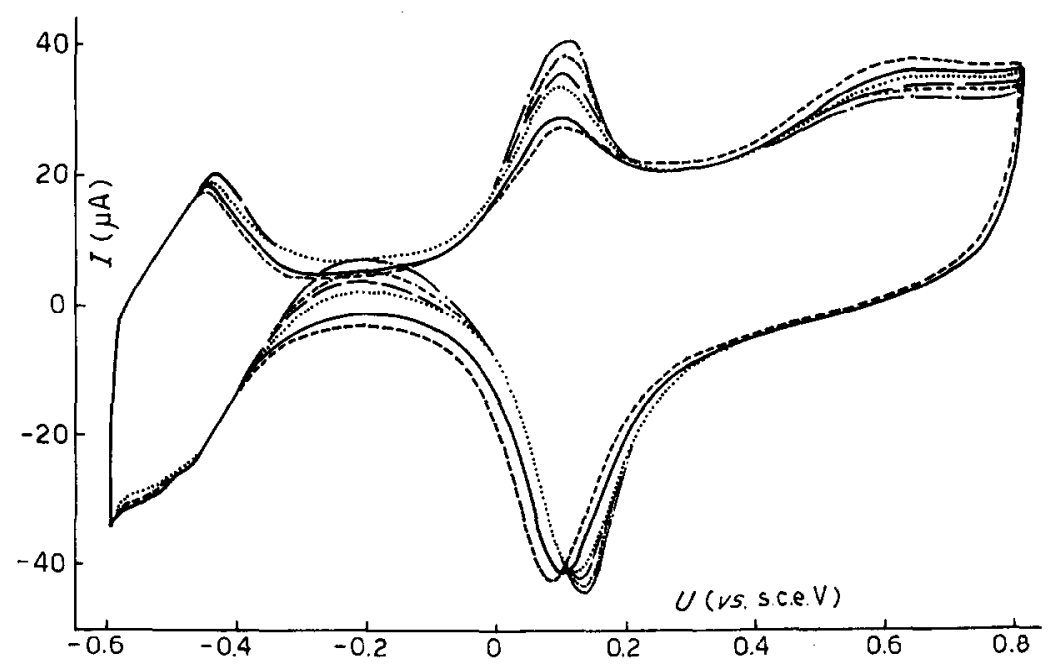

Fig. 11. Potentiodynamic $U / I$ profiles corresponding to TPS (0.03 $M$ glucono- $\delta$-lactone solution) run at

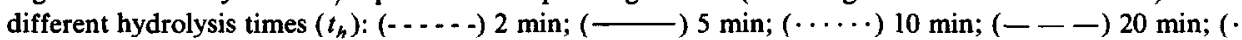
- - .) $30 \mathrm{~min} ;(\cdot-\cdot-\cdot) 50 \mathrm{~min}$.

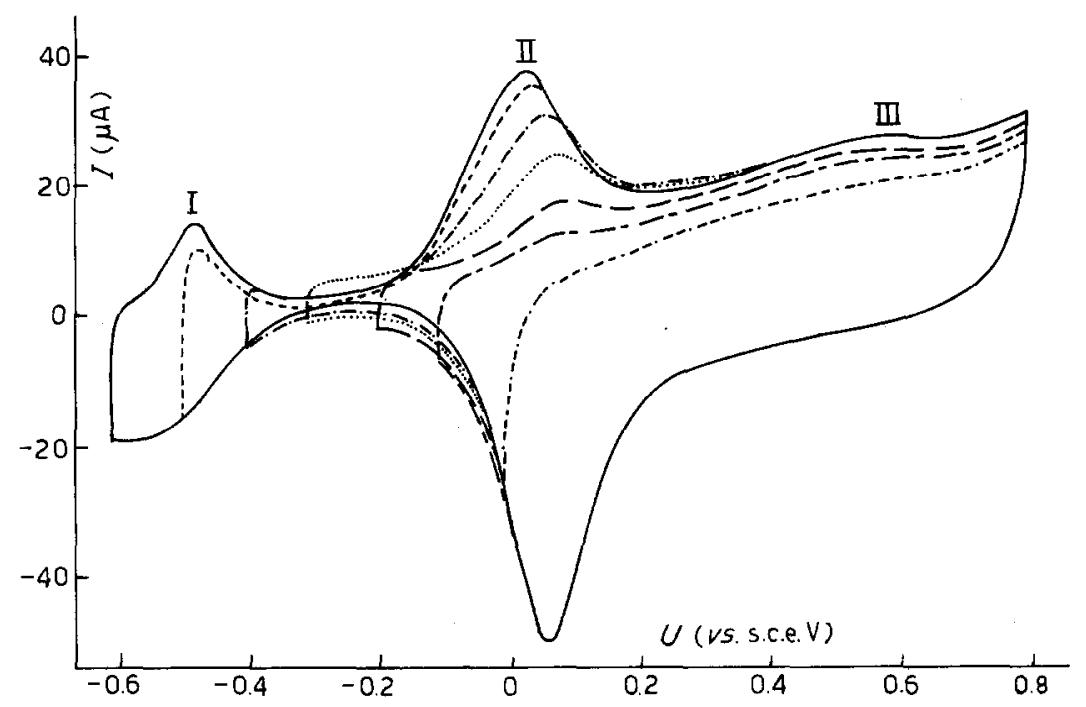

Fig. 12. Repetitive TPS ( $0.03 M$ fructose solution) run in the following potential ranges: $(-)-0.6 \mathrm{~V}$ to $0.8 \mathrm{~V} ;(-\ldots .)-.0.5 \mathrm{~V}$ to $0.8 \mathrm{~V} ;(\cdot-\cdot-\cdot)-0.4 \mathrm{~V}$ to $0.8 \mathrm{~V} ;(\cdots \cdots)-0.3 \mathrm{~V}$ to $0.8 \mathrm{~V} ;(---)$ $-0.2 \mathrm{~V}$ to $0.8 \mathrm{~V} ;(-)-0.1 \mathrm{~V}$ to $0.8 \mathrm{~V} ;(\cdot--\cdot) 0.0 \mathrm{~V}$ to $0.8 \mathrm{~V}$. 
time-dependence of the $U$ versus $I$ displays run in the presence of glucono- $\delta$-lactone (Fig. 11) is attributed to the different hydrolysis time $\left(t_{h}\right)$ involved in the various runs. Thus, both the height of peak II and the current in the potential range of peak IV increase when $t_{h}$ increases and the relative gradual change of the $U$ versus $I$ profile suggests an increasing contribution of the electro-oxidation of gluconic acid as the hydrolysis reaction proceeds.

\section{$U$ versus I profiles run in the presence of fructose}

In $\mathrm{D}(-)$ fructose the reaction between the carbonyl group at carbon atom 2 and the hydroxyl group yields either a five-member ring (furanose form) or a six-member ring (piranose form). The $U$ versus $I$ displays obtained in the presence of fructose are similar to those resulting for gluconic acid (Fig. 12). They show two peaks and a plateau during the positive-going potential sweep and a broad anodic current region following the oxygen reduction.

As the cathodic switching potential is fixed at more positive values, the oxidation current, particularly in region II, becomes lower. Thus, processes occurring in region I are probably related to those corresponding to region II.

The changes of the $U$ versus $I$ profiles in the $\mathrm{H}$-adatom region, the current response at $U_{a d}$ and the influence of $t_{a d}$ on the heights of current peaks corresponding to fructose electro-oxidation are directly comparable to those already described for the gluconic acid-containing electrolyte.

\section{$U / I$ displays obtained in the presence of xylose and arabinose}

Aldopentoses such an xylose and arabinose have three asymmetric carbon atoms, and therefore they can form eight stereoisomers of which D-xylose and D-arabinose differ from each other in the position of the $\mathrm{OH}$ associated with $\mathrm{C}$ atoms 2 and 3. The position of the $\mathrm{OH}$ groups related to the asymmetric carbon atoms in the $\mathrm{D}$-arabinose molecule is similar to that of the glucose molecule. To establish the influence of the position of the $\mathrm{OH}$-groups as well as the number of carbons of the ring on the electrochemical behaviour of monosaccharides with hemiacetalic structure, runs with added solutions of D-xylose and D-arabinose were made.

The potentiodynamic $U$ versus $I$ display obtained in the presence of arabinose (Fig. 13) is comparable to that of glucose. It exhibits three anodic current peaks (I,II,III) during the positive-going potential scan and a large anodic peak $\mathrm{V}$ and a hump IV during the reverse potential sweep. The position of the anodic complex peak $\mathrm{V}$ is mainly influenced by the electrolyte composition, as observed in the case of glucose [26,27]. Similar results are obtained in the presence of xylose. These results suggest that no great modification of the $U$ versus $I$ profile should be expected neither due to the different position of the $\mathrm{OH}$ groups in the molecule nor to the different size of the O-carbon ring. 


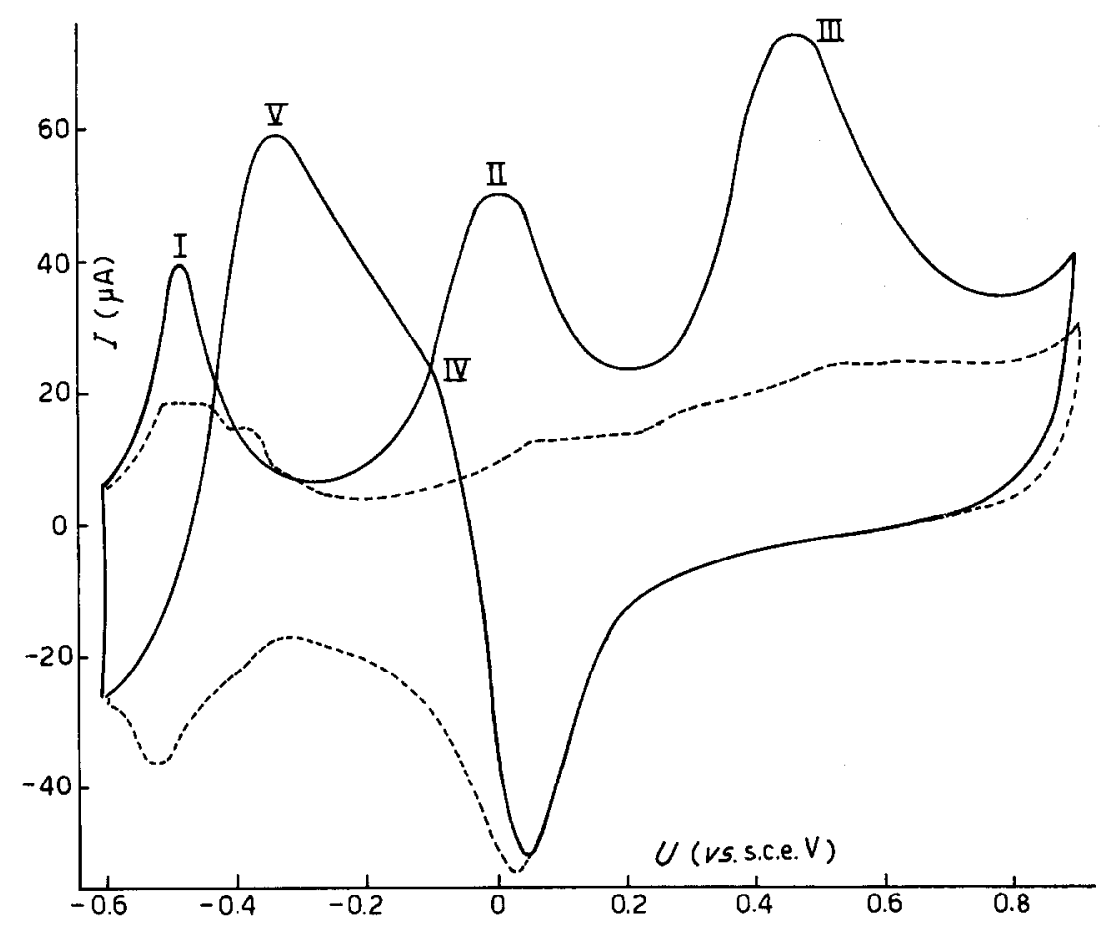

Fig. 13. Potentiodynamic $U / I$ profiles run in a $0.03 M$ arabinose solution (potential/time perturbation program shown in Fig. 1a, $U_{a d}=-0.6 \mathrm{~V}, t_{a d}=10 \mathrm{~s}$ ). Dotted line corresponds to the base electrolyte.

\section{$U / I$ profiles run in the presence of inositol}

The molecule of inositol differs from that of glucose only in its cyclic structure. Both molecules contain the same number of carbon atoms as well as $\mathrm{OH}$ groups. Though both structures correspond to a six-member ring, glucose is a heterocyclic ring while inositol is a carbocyclic one.

During the positive-going potential scan (Fig. 14), the electro-oxidation current covers a potential range (II,III) which coincides with the potential range related to the electroformation of the $\mathrm{Pt}(\mathrm{O})_{a d}$ layer. On the other hand, the reverse potential scan shows a slight increase in the cathodic current at potentials exceeding that of the O-electrodesorption from Pt. Figure 14 shows the current decrease in region II when the cathodic switching potential is increased. Thus, the electro-oxidation processes occurring in regions II-III appear related to the processes corresponding to region I. However, as was observed in the case of gluconic acid, the transient current recorded at $U_{a d}$ exhibit practically no appreciable current peak. Only at higher temperatures and higher concentrations do currents corresponding to regions III and IV appreciably increase. 


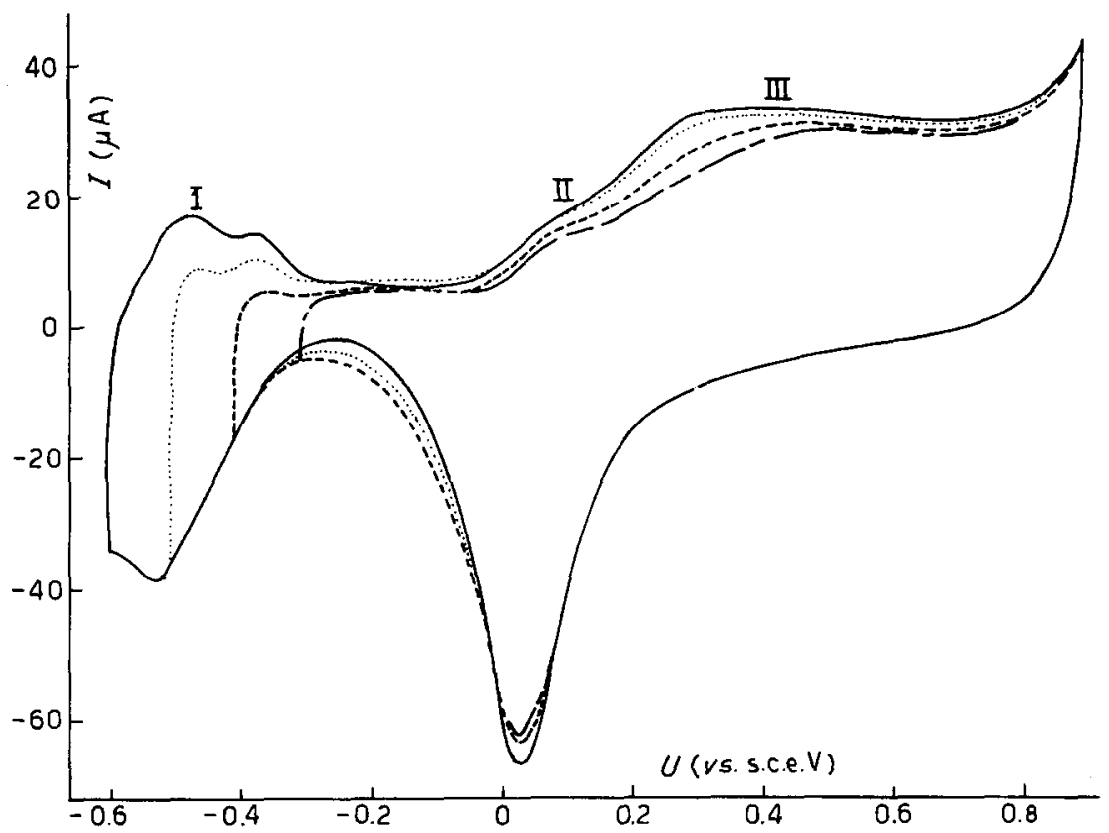

Fig. 14. Repetitive $U / I$ profiles ( $0.03 M$ inositol solution) run in the following potential ranges: ( $-0.6 \mathrm{~V}$ to $0.9 \mathrm{~V} ;(\cdots \cdots)-0.5 \mathrm{~V}$ to $0.9 \mathrm{~V} ;(-\ldots .-)-0.4 \mathrm{~V}$ to $0.9 \mathrm{~V} ;(---\ldots)-0.3 \mathrm{~V}$ to $0.9 \mathrm{~V}$.

$U / I$ profiles run in the presence of $\alpha-D-$ glucose-1-phosphate dipotassium salt $\left(G-I-P-K_{2}\right)$

Taking into account that glucose, xylose and arabinose show similar electrochemical responses and considering that the hemiacetalic structure is a common feature of these monosaccharides, G-1-P-K 2 (Cori ester) was electrooxidized to determine the influence of the esterification of the OH-group in the C-1 position on the electrochemical reaction.

The $U / I$ profile obtained in G-1-P-K $\mathrm{K}_{2}$ is remarkably different from that of glucose (Fig. 15). The electro-oxidation current is very small and the shape of the $U / I$ profile approaches that of the Pt|electrolyte interphase in the absence of the organic molecule. These results are of interest in the following discussion of a correlation of electrochemical kinetic behaviour of monosaccharides.

\section{DISCUSSION}

Gluconic acid has been considered as the main reaction product of glucose electro-oxidation by several authors $[15,24,25]$, although the available data on its electrochemical response are rather scarce and incomplete. On the other hand, it has been suggested that the electrochemical behaviour of gluconic acid is to some extent similar to that of glucose except at high anodic values [16,17]. The present results on 


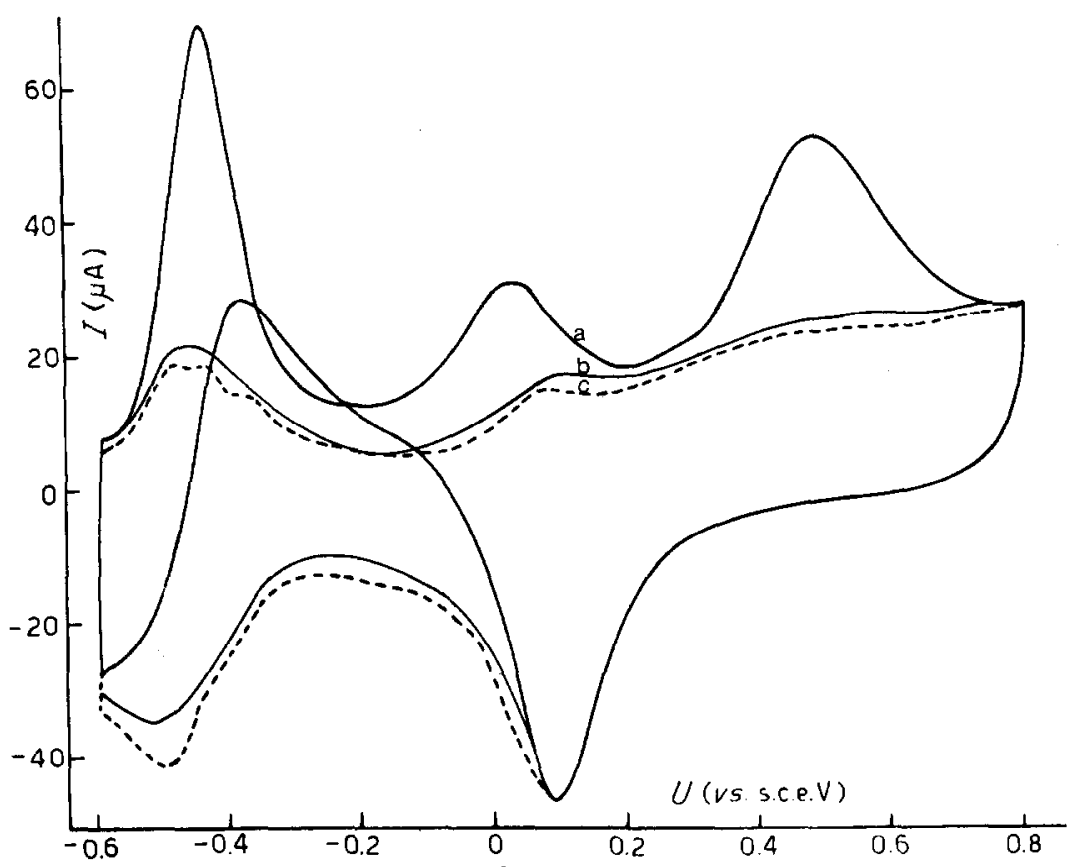

Fig. 15. Potentiodynamic $U / I$ profiles run in: (a) $0.03 M$ glucose solution; (b) $0.03 M \mathrm{G}-1-\mathrm{P}-\mathrm{K}_{2}$ solution; (c) base electrolyte (potential/time perturbation program shown in Fig. 1a, $U_{a d}=-0.6 \mathrm{~V}, t_{a d}=10 \mathrm{~s}$ ).

the electro-oxidation of these compounds, in contrast with previous data show remarkable differences in the corresponding potentiodynamic $U$ versus $I$ profiles (Figs. 2 and 3). Furthermore, the first potentiodynamic sweep in the negative potential direction covering the hydrogen ad-atom region shows that the anodic current contribution is large only in the solutions containing glucose. During the following potential scans the electrode surface becomes progressively blocked by the organic substances, both in the presence of either glucose or gluconic acid. These results suggest that some intermediate is adsorbed on the electrode and remains there at low potentials. This intermediate, however, may diffuse into the solution through its participation in an electroadsorption/desorption process, yielding glucono- $\delta$-lactone, a product which has been detected during the electro-oxidation of glucose in the low potential region [25]. The intermediate product which remains adsorbed, can be electro-oxidized after the electrode potential exceeds a certain threshold potential which is associated with the activation of the electrode [27]. In addition, the anodic charge measured at the adsorption potential $U_{a d}$, for glucose, is much greater than that for gluconic acid (Fig. 6).

On the other hand, the dependence of the open-circuit potential $[34,35]$ on the nature of the organic substance in solution is coherent with the potentiodynamic data. Thus, in the presence of glucose, the potential attains a value which is in the potential range of the hydrogen adatoms and the corresponding relaxation time $(\tau)$ 
depends on glucose concentration [34]. This relaxation time can be assigned to the time required for electroreducing the $\mathrm{PtO}$ layer, probably through a mechanism involving a mixed electrochemical potential such as that usually found in corrosion processes. In the gluconic acid solutions the fact that there is no appreciable change in potential under open-circuit conditions would indicate that under those conditions there is neither electroadsorption/dehydrogenation of this molecule nor PtO electroreduction.

The different electrochemical behaviour of glucose and gluconic acid can be correlated with their structural characteristics. Thus, gluconic acid results from the oxidation of the aldehydic group of glucose and presents a structure differing basically from that of glucose, both in the open structure and in the presence of the carboxylic group. To distinguish between the influence of either the open structure or the carboxylic group, the comparative electrochemical data obtained with the various compounds of different structures are relevant. Neither the six-member ring with six OH-groups (inositol) (Fig. 14) nor the heterocyclic one with an O-member (fructose) (Fig. 12) exhibit a similar behaviour to that of glucose. Only those molecules involving heterocyclic forms with a hemiacetalic structure, show an electrochemical response similar to that of dextrose (Fig. 13). This similarity is independent of the size of the ring (arabinose) and the position of the OH-group of the asymmetric carbon atoms (xylose). Henceforth, this points out that the hemiacetalic structure is actually responsible of the electrochemical behaviour of glucose in the hydrogen adatom potential region, and the electroadsorption taking place at those potentials is due to the great reactivity of the hydrogen atom related to the hemiacetalic group. In addition, runs made with G-1-P-K $\mathrm{K}_{2}$ proved that when the hemiacetalic group is esterified, the corresponding $U$ versus $I$ profiles are different from those of glucose. This fact can be explained in terms of a shielding of the active hydrogen atom by the phosphate group whose size exceeds that of the hemiacetalic group. Therefore, the esterification of the hemiacetalic group hinders in this case the electroadsorption and oxidation of the molecule in the potential range of the hydrogen adatoms.

The comparative kinetic data gives further support to the appearance of glucono$\delta$-lactone and gluconic acid as reaction products [24] at low potentials, through firstly, a dehydrogenation of the hemiacetalic form of glucose, followed by desorption and hydrolysis of the electroadsorbed species, as has been postulated previously [25].

The interpretation of the reactivity of the different carbohydrates in terms of the hydrogen atoms bound to the carbon atom in the 1-position is also consistent with the influence of temperature on the characteristies of the $U$ versus $I$ profiles. Thus, when temperature is increased, the $\mathrm{p} K$ values of the monosaccharides increase, showing that proton ionization in the 1-position is favoured. Hence the electroadsorption process and the electrode blockage occur faster. In addition, the temperature increase may produce structural changes of the molecules. This appears to be the case in solutions containing xylose, where the spectrophotometric absorbance of these solutions increases accordingly with the temperature, a result which was 
explained through the ring opening [36]. Therefore, some of the changes observed in the electrochemical response of glucose solutions when temperature is increased can be, in part, also assigned to the structural rearrangement of the molecule around the anomeric carbon atom of glucose which occurs via the open structure of the dextrose. Finally, as was reported previously [20,26,27,37], changes in the electrolyte composition including $\mathrm{pH}$ can directly affect the equilibrium between the possible reactive forms.

Therefore, a correlation between the rate of electro-oxidation and molecular structure can be derived for the conversion of carbohydrates and derivatives. This correlation can be put forward in terms of the availability of the reacting hydrogen atom bound to the hemiacetalic group. For this purpose, the heights of current peaks $\mathrm{I}$ and $\mathrm{V}$ can be taken as a measure of the electro-oxidation capability of the molecule. In this case, the following order results for constant electrolyte composition, temperature and electrode pretreating: Glucose, arabinose, xylose $>$ gluconic acid, fructose $>$ inositol, glucono- $\delta$-lactone, G-1-P-K .

From the structural standpoint the electrochemical reactivity of the $\mathrm{H}$-atom in the cyclic monosaccharides is enhanced by the $\mathrm{O}$-atom in $\mathrm{C}-1$ and $\mathrm{C}-(\mathrm{n}-1)$ carbon.

The electro-oxidation order of the different carbohydrates and derivatives resembles to some extent the electrochemical conversion order already established for the electro-oxidation of alcohols, aldehydes and aliphatic organic acids, which in this case is directly proportional to the hydrogen atoms bound to the $\alpha$-carbon.

The conclusions from the comparative electro-oxidation of different carbohydrates in terms of the electrochemically reactive hemiacetalic group gives further support to the general reduction pathway advanced previously for electro-oxidation of glucose on platinum in different electrolytes [27].

\section{REFERENCES}

1 J.R. Rao and G. Richter, Naturwissenschaften, 61 (1974) 200.

2 J.R. Rao, G. Richter, F. von Sturm, E. Weidlich and M. Wenzel, BioMed. Eng., 9 (1974) 98.

3 B.Y.C. Wan and A.C.C. Tseung, Med. Biol., Eng., 12 (1974) 14.

4 E. Weidlich, G. Richter, F. Von Sturm and J.R. Rao, Biomater. Med. Devices Artif. Organs, 4 (1976) 277.

5 E.J. Lahoda, C.C. Liu and L.B. Wingard, Int. Soc. Energy Convers., 7th Eng. Conf. Proc. 1972, p. 740.

6 S.K. Wolfson, L.B. Wingard, C.C. Liu and S.J. Yao, Biomedical Applications of Immobilized Enzymes and Proteins, Plenum Press, New York, 1977, Vol. 1, p. 377.

7 K.W. Chang, S. Aisenberg, J.S. Soeldner and J.M. Hiebert, Trans. Am. Soc. Artif. Intern. Organs, 19 (1973) 352.

8 S.P. Bessman and R.D. Schultz, Trans. Am. Soc. Artif. Intern. Organs, 19 (1973) 361.

9 J.R. Rao, G.J. Richter, F. Von Sturm and E. Weidlich, Bioelectrochem. Bioenerg., 3 (1976) 139.

10 J.R. Rao, G.J. Richter, G. Luft and F. Von Sturm, Biomater. Med. Devices Artif. Organs, 6 (1978) 127.

11 V. Gebhardt, G. Luft, G. Richter and F. Von Sturm, Bioelectrochem. Bioenerg., 5 (1978) 607.

12 D. Gough, F. Anderson, J. Giner, C. Colton and J.S. Soeldner, Anal. Chem, 50 (1978) 941.

13 C.C. Liu, L.B. Wingard, Jr., S.K. Wolfson, Jr., S.J. Yao, A.L. Drash and J.G. Shiller, Bioelectrochem. Bioenerg., 6 (1979) 19.

14 E.A. Disalvo and H.A. Videla, 6th Intern. Bioelectrochem. and Bioenerg. Symp. Kiryat Anavim, Israel, 1981, Abstracts, p. 20. 
15 M.L. Rao and R. Drake, J. Electrochem. Soc., 116 (1969) 335.

16 S.J. Yao, A.J. Appleby, A. Geisel, H.R. Cash and S.K. Wolfson Jr., Nature (London)., 224 (1969) 921.

17 J. Giner and P. Malachesky, Proceedings of the Artificial Heart Program Conference, Washington D.C., 1969 , p. 839.

18 H. Lerner, J. Giner, J.S. Soeldner and C. Colton, J. Electrochem. Soc., 126 (1979) 237.

19 L. Marincic, J.S. Soeldner, V. Giner and C. Colton, J. Electrochem. Soc., 126 (1979) 43.

20 M.F.L. de Mele, H.A. Videla and A.J. Arvia, 32nd I.S.E. Meeting, Dubrovnik/Cavtat, 1981.

21 E.M. Skou, 27th I.S.E. Meeting, Zürich, Ext. Abstr., 1976, p. 279.

22 E.M. Skou, 29th I.S.E. Meeting, Budapest, Ext. Abstr., 1978, p. 668.

23 E.M. Skou, Electrochim. Acta, 22 (1977) 313.

24 S. Ernst, J. Heitbaum and C. Hamann, J. Electroanal. Chem., 100 (1979) 173.

25 S. Ernst, J. Heitbaum and C. Hamann, Ber. Bunsenges. Phys. Chem., 84 (1980) 50.

26 M.F.L. de Mele, H.A. Videla and A.J. Arvia, 6th Int. Bioelectrochem. Bioenerg. Symp. Kiryat Anavim, Israel, 1981, Abstracts, p. 4.

27 M.F.L. de Mele, H.A. Videla and A.J. Arvia, J. Electrochem. Soc., in press

28 K. Ito, S. Ikeda, H. Yamaguchi, K. Ito and T. Kondo, Denki Kagaku Oyobi Kogyo Butsuri Kagaku, 46 (1978) 141.

29 N.N. Nikolaeva, O.A. Khazova and Y.B. Vasilev, Sov. Electrochem., 16 (1981) 1022.

30 S. Gilman, Trans. Faraday Soc. 62 (1966) 466.

31 S. Gilman, Trans. Faraday Soc. 62 (1966) 481.

32 M.E. Folquer, J.O. Zerbino, N.R. de Tacconi and A.J. Arvía, J. Electrochem. Soc., 126 (1979) 593.

33 E.M., Skou, Thesis, Lyngby, Denmark, 1974.

34 M.F.L. de Mele, H.A. Videla, An. Asoc. Quim. Argent. 69 (1981) 269.

35 W. Hauffe and J. Heitbaum, Electrochim. Acta, 23 (1978) 299.

36 J. Christensen, J. Rytting and M. Izatt, J. Chem. Soc. B, (1970) 1646.

37 A.W. Fonds and J.M. Los, J. Electroanal. Chem., 36 (1972) 479. 